\title{
Effect of neonatal hyper bilirubinemia on brain stem auditory evoked response
}

\author{
Kosam A. ${ }^{1}$, Khunte M. ${ }^{2}$, Panigrahi D. ${ }^{3}$ \\ ${ }^{1}$ Dr. Ajay Kosam, Professor, ${ }^{2}$ Dr. Madhuri Khunte, Assistant Professor, ${ }^{3}$ Dr. Deepak Panigrahi, Senior Resident, all \\ authors are affiliated with Department of Paediatrics, Bharat Ratna Late Shri Atal Bihari Vajpayee Memorial \\ Government Medical College (BRLSABVMGMC), Rajnandgaon, Chhattisgarh, India.
}

Corresponding Author: Dr. Madhuri Khunte, F-6 Hospital Colony, Basantpur, Rajnandgaon (C.G.) E- mail: drdeepak085@gmail.com

\begin{abstract}
Introduction: Neonatal hyperbilirubinemia is a major cause of morbidity in neonates. The long term neurological sequel can be prevented and reversed by timely and aggressive management of hyperbilirubinemia. Brain stem auditory evoked response (BAER) is an assessment tool to help predictimpeding bilirubin neurotoxicity. This study was conducted to evaluate the effect of hyperbilirubinemia on auditory system of newborn and the effect of therapy on BAER. Materials and Methods: In this case control study, 50 term neonates with hyperbilirubinemia (total serum bilirubin $>15 \mathrm{mg} / \mathrm{dl}$ ) were included in the study group and 25 normal term neonates were taken as controls. Baseline BAER was recorded in study group before therapy and after therapy. Results were compared with controls and intra group comparison was also done. Continuous data with normal distribution was analysed by student t-test, and categorical data was analysed using chi-square test. Results: Most common BAER abnormality noted in jaundiced neonates was prolonged latency of wave V (42\%) and prolonged inter wave interval I-V (32\%). Significant increase in the absolute latencies of waves III and V was noted in hyperbilirubinemic neonates as compared to controls $(\mathrm{p}<0.05)$. I-III and I-V inter-peak latencies were also significantly prolonged in neonates with hyper bilirubinemia $(\mathrm{p}<0.05)$. There was significant improvement in the latency of wave III and wave V, I - III inter- peak latency and I - V inter-peak latency after treatment $(\mathrm{P}<0.05)$. Conclusion: Results of our study demonstrate the importance of early ABR screening as an efficient tool for monitoring the neonates at risk of bilirubin neurotoxicity. Diagnosing the early changes in ABR caused by hyperbilirubinemia before appearance of clinical abnormality will help prevent bilirubin neurotoxicity.
\end{abstract}

Keywords: Brainstem auditory evoked response, Hyperbilirubinemia, Latency, Neurotoxicity, Waves.

\section{Introduction}

Neonatal indirect hyperbilirubinemia is a common problem in newborns [1]. Neurological problems such as athetoid dystonic cerebral palsy, hearing loss, gaze palsy, developmental delay and impairment of intelligence due to bilirubin encephalopathy are serious problems [2]. Chronic bilirubin encephalopathy leads to partial to complete sensorineural deafness.

Despite progress in understanding the process of bilirubin neurotoxicity and advances in technology to measure early effects of bilirubin in brain, the question of what is the safe level of bilirubin concentration and safe duration of exposure has not been fully explained. The critical bilirubin level that results in neurotoxicity is not clear.

Manuscript received: $4^{\text {th }}$ February 2019

Reviewed: $14^{\text {th }}$ February 2019

Author Corrected: $19^{\text {th }}$ February 2019

Accepted for Publication: $23^{\text {rd }}$ February 2019
In view of these uncertainties new assessment tools have been sought to help predict impending bilirubin neurotoxicity. Because the auditory pathway of neonates is particularly vulnerable to insult from bilirubin, Brain stem auditory evoked response (BAER) has been suggested as a tool that could identify and predict early effects of hyperbilirubinemia on nervous system [3].

Hyperbilirubinemia has been associated with abnormalities in brain stem auditory evoked response (BAER). The conventional auditory brainstem response (ABR) is recognized as the most objective method of evaluating the auditory system in neonates and infants [4]. The ABR measures activity from auditory nerve up to the level of brainstem stimulated by acoustic stimuli which are typically clicks for the purpose of screening. 


\section{Original Research Article}

The response which reflects synchronous activation of primary onset type neurons in the auditory system occurs within 5 to 6 milliseconds following high intensity acoustic stimuli as a series of major peaks in waveforms [5]. Wave forms are labelled by Roman numerals with wave $\mathrm{V}$ considered to be the most robust at low stimulus intensities. ABR is not a test of hearing but it assesses the neural integrity of the auditory pathway up to the brainstem [5].

Several studies have revealed abnormal ABR results in infants with hyperbilirubinemia. Abnormality reported was an increase in the wave's latency. ABR abnormality in these infants indicates early bilirubin ototoxicity [6]. The present study was conducted to evaluate the effects of hyperbilirubinemia on Auditory Brainstem Response in newborn and to assess the reversibility of ABR.

\section{Material and Methods}

\section{Aim and objectives}

1. To determine the brainstem auditory evoked response (BAER) abnormalities in neonates with indirect hyperbilirubinemia.

2. To evaluate the reversibility of abnormal BAER after therapy.

The present study is a case control study carried out on term neonates admitted in SNCU of Bharat Ratna Late Shri Atal Bihari Vajpayee Memorial Government Medical College Hospital, Rajnandgaon, Chhattisgarh from January 2018 to December 2018.

50 term neonates with hyperbilirubinemia were included in the study group and 25 normal term neonates were taken as control group. Infants with hyperbilirubinemia were divided into 2 groups.Group I included neonates with bilirubin level $15-20 \mathrm{mg} / \mathrm{dl}$ and Group II consisted of infants with bilirubin level $>20 \mathrm{mg} / \mathrm{dl}$. Baseline BAER was recorded in study group before therapy and after therapy and results were compared with controls and intra group comparison was also done.

Neonates with craniofacial anomalies, preterm babies, low birth weight babies, neonates with exposure to ototoxic medications, birth asphyxia, acute bacterial meningitis, intra-uterine infections were excluded from the study.

Anthropometric measurements were taken at the time of admission. A detailed history with emphasis on the onset of jaundice, risk factors present, maternal drugs (oxytocin, diazepam, promethazine), maternal risk factors (age $>24$ years, diabetes, order of gestation, oral contraceptive use at time of conception), previous sibling history, feeding history, starting of phototherapy. All neonates were examined for bruises, cephalhematoma, scalp injuries, liver and spleen enlargement. Following mandatory investigations were done in all babies: hematocrit, peripheral smear, reticulocyte count, total and conjugated bilirubin, blood group of mother and baby and direct Coomb's test. TSH level and septic screen was done whenever applicable.

Neonates with hyperbilirubinemia were managed as per AAP guidelines [7].

The equipment used for assessing the BAER was GSI ABR/ASSR system. Three silver coated electrodes were used to record ABR. One electrode was placed on infants forehead (non- inverting) known as active electrode, second electrode - inverting or reference electrode was placed on test ear mastoid and third electrode - ground electrode was placed on non test ear mastoid.

BAER recording was done in sound proof room. Neonates were evaluated during normal sleep or soon after feeding. Click stimulus of 0.1 millisecond duration at the rate of 10-40 clicks/second was presented through ear phones.

The recording was obtained as graph with amplitude (micro-volts) on the ordinate and time (milliseconds) on abscissa. It consists of five to seven waves or peaks appearing within 8 or 10 milliseconds. For proper interpretation of the BERA graph, the different waves especially the waves I, III, V have to be accurately identified. The waves are generated at following points of the auditory pathway between the cochlea and the brain stem [8].

\section{Wave Site of Neural Generator}

1. Cochlear Nerve (Distal end)

2. Cochlear Nucleus

3. Superior Olivary Complex

4. Lateral Lemniscus

5. Inferior Colliculus

6. Not known

7. Not known

Following parameters were studied - absolute peak latency of ABR waveforms, amplitude of wave, interwave latency interval, latency intensity function of wave $\mathrm{V}$. 


\section{Original Research Article}

Statistical analysis- Results are expressed as mean \& SD. Numerical data were analyzed by SPSS 21.0 Version. Continuous data with normal distribution was

analysed by student t-test, and categorical data was analysed using chi-square test.

\section{Results}

Table-1: Demographic profile of patients in two groups.

\begin{tabular}{|c|c|c|}
\hline Patient Characteristics & Cases $(\mathbf{n = 5 0})$ & Controls $(\mathbf{n}=\mathbf{2 5})$ \\
\hline Birth weight (grams) & $2425 \pm 377$ & $2510 \pm 400$ \\
\hline Age (days) & $4.3 \pm 1.2$ & $4.0 \pm 1.3$ \\
\hline Sex & & $15(60 \%)$ \\
Male & $28(56 \%)$ & $10(40 \%)$ \\
Female & $22(44 \%)$ & \\
\hline
\end{tabular}

The two groups were similar in respect to age at entry into study, birth weight and sex.

Table-2: Cause of hyperbilirubinemia.

\begin{tabular}{|c|c|}
\hline Etiology of hyperbilirubinemia & Cases \\
\hline ABO Incompatibility & $22(44 \%)$ \\
\hline Rh Incompatibility & $6(12 \%)$ \\
\hline Idiopathic & $19(38 \%)$ \\
\hline Cephalhematoma & $3(6 \%)$ \\
\hline
\end{tabular}

ABO incompatibility was the most common cause of hyperbilirubinemia (44\%) followed by Idiopathic (38\%), Rh incompatibility (12\%) and cephalhematoma (6\%).

Table-3: ABR abnormality in neonates with hyperbilirubinemia

\begin{tabular}{|c|c|}
\hline ABR abnormality & Cases \\
\hline Prolonged latency wave V & $42 \%$ \\
\hline Prolonged inter wave interval I - V & $32 \%$ \\
\hline Prolonged inter wave interval I - III & $30 \%$ \\
\hline Diminished amplitude of wave V & $22 \%$ \\
\hline
\end{tabular}

Most common BAER abnormality noted was prolonged latency of wave V (42\%) followed by prolonged inter wave interval I - V (32\%), prolonged inter wave interval I - III (30\%) and diminished amplitude of wave V (22\%).

Table-4: Measurements of ABR absolute latencies and inter peak latencies in neonates with hyperbilirubinemia and normal controls.

\begin{tabular}{|c|c|c|c|}
\hline ABR Parameters & Controls & Hyperbilirubinemia & P value \\
\hline Latency (ms) & & & $\mathrm{P}>0.05$ \\
\hline I & $1.30 \pm 0.10$ & $1.32 \pm 0.11$ & $\mathrm{P}<0.05$ \\
\hline III & $3.42 \pm 0.10$ & $4.24 \pm 0.24$ & $\mathrm{P}<0.05$ \\
\hline V & $5.70 \pm 0.22$ & $7.9 \pm 0.60$ & $\mathrm{P}<0.05$ \\
\hline Intervals (ms) & & & $\mathrm{P}>0.05$ \\
\hline I - III & $2.10 \pm 0.13$ & $2.92 \pm 0.22$ & $\mathrm{P}<0.05$ \\
\hline III - V & $2.33 \pm 0.26$ & $2.40 \pm 0.28$ & $5.36 \pm 0.34$ \\
\hline I-V & $4.42 \pm 0.24$ & & \\
\hline
\end{tabular}

There is significant increase in latency of wave III and V in hyperbilirubinemia group as compared to control group $(p<0.05)$ but wave I latency was comparable between two groups $(p>0.05)$. 
I-III inter-peak latency and I-V inter-peak latency was significantly longer in hyperbilirubinemia group as compared to controls $(\mathrm{p}<0.05)$ but III-V inter-peak latency was comparable between two groups $(\mathrm{p}>0.05)$. Thus wave $\mathrm{V}$ is most commonly affected by hyperbilirubinemia.

Table-5: Measurements of ABR absolute latencies and inter peak latencies before and after treatment

\begin{tabular}{|c|c|c|c|}
\hline ABR Parameters & Before treatment & After treatment & P value \\
\hline Latency (ms) & & & \\
\hline I & $1.32 \pm 0.11$ & $1.31 \pm 0.11$ & $\mathrm{P}>0.05$ \\
\hline III & $4.24 \pm 0.24$ & $3.46 \pm 0.09$ & $\mathrm{P}<0.05$ \\
\hline V & $7.9 \pm 0.60$ & $5.73 \pm 0.25$ & 0.05 \\
\hline Intervals (ms) & & & $\mathrm{P}<0.05$ \\
\hline I - III & $2.92 \pm 0.22$ & $2.34 \pm 0.15$ & $\mathrm{P}>0.05$ \\
\hline III - V & $2.40 \pm 0.28$ & $2.36 \pm 0.26$ & $\mathrm{P}<0.05$ \\
\hline I-V & $5.36 \pm 0.34$ & $4.47 \pm 0.22$ & $\mathrm{P}$ \\
\hline
\end{tabular}

There was significant improvement in the latency of wave III and wave V after treatment $(\mathrm{P}<0.05)$.I - III inter- peak latency and I - V inter-peak latency significantly improved after treatment $(\mathrm{P}<0.05)$

\section{Discussion}

Significant increase in unconjugated bilirubin in neonates coupled with other risk factors like sepsis, acidosis, hypoxia, hypoglycaemia and hypothermia predisposes to bilirubin neurotoxicity. Deposition of unconjugated and free bilirubin in specific regions of brain, especially the basal ganglia, pons and cerebellum causes encephalopathy [9- 10]. Neonatal hyperbilirubinemia and its adverse effects on sensory and motor system is still a major problem despite advances in medical field. Reason for this problem is multifactorial like early hospital discharge of neonates, lack of adequate knowledge about neurological effects of hyperbilirubinemia, and lack of follow-up of these high risk neonates [11].

Various newer techniques have been used to predict impending bilirubin encephalopathy. Infant cry characteristics analysis, nuclear magnetic resonance imaging, nuclear magnetic resonance spectroscopy, diffusion weighted NMR imaging and brainstem auditory evoked response have been evaluated as tools in this regard [12-14 ].

Toxic effects of bilirubin at cellular level are due to interruption of normal neurotransmission, mitochondrial dysfunction, cellular and intracellular membrane impairment and interference with enzyme activity [15]. The neonatal auditory system is very sensitive to high levels of bilirubin [15]. BAER abnormalities in hyperbilirubinemia may be due to neurotransmission abnormality and inhibition of neurotransmitter release. Changes in membrane potential in cells involved in synapse transmission have also been theorized as the cause of bilirubin neurotoxicity causing ABR abnormalities [16]. ABR waves I, III and V with latency values have physiological and clinical importance. Changes in latency values of these waves indicate disturbances in the auditory brainstem function [16]. Wave I originates from spiral ganglion cells of the auditory nerve that connect to the cochlea. Waves III and $\mathrm{V}$ originate from lower and upper brainstem areas [17].

In our study absolute latencies of waves III and V are significantly prolonged in neonates with hyperbilirubinemia as compared to normal controls. Wave I latency in neonates with hyperbilirubinemia was comparable to control group. This indicates an abnormality in the central auditory pathway. Similar observation was noted by other studies [18-19].

I-III and I-V inter-peak intervals were significantly prolonged in our study pointing towards delayed brainstem conduction time suggesting synapses as the primary target for bilirubin effects. Our study demonstrated that wave $\mathrm{V}$ absolute latency was predominantly affected by hyperbilirubinemia with consequent increase in $\mathrm{I}-\mathrm{V}$ inter-peak latency suggesting that rostral regions of the brainstem are more sensitive to an increase in bilirubin levels than caudal region [18-19]. Our study however did not demonstrate any significant difference in wave I absolute latency between two groups. This could be due to the noninvolvement of the cochlear nerve [20]. In severe 
hyperbilirubinemia an increase in wave I absolute latency can also be demonstrated [21-23]. The results of this study demonstrate that auditory brainstem nucleuses are the main target of bilirubin toxicity. Thus Oto-acoustic Emission (OAE) test for hearing screening programs for high risk neonates before discharge from the hospital may be inadequate [23]. The central auditory impairment observed in our study can have important clinical implications in terms of long term neurodevelopment outcome.

Significant improvement in the latency of wave III, wave V, I - III inter- peak latency and I-V inter-peak latency was observed in our study after therapy. Similar observation has been noted by other studies [18-19, 24]. This indicates that these early ABR changes are transient and effects of hyperbilirubinemia on central auditory system are reversible with timely intervention.

However persistence of ABR abnormalities even after discharge from the hospital has been noted by some studies which indicate axonal degeneration and loss of myelin and highlight the importance of rapid treatment [25].

This study emphasizes on the importance of early ABR changes with special attention to central impairment after neonatal hyperbilirubinemia and rapid intervention to prevent irreversible damage.

Neonates in our study had no co-existing pathological process that could have altered the ABR responses. These observed changes in latency values are attributed to hyperbilirubinemia.

\section{Conclusion}

Results of our study demonstrate the importance of early ABR screening as an efficient tool for monitoring the neonates at risk of bilirubin neurotoxicity. Diagnosing the early changes in ABR caused by hyperbilirubinemia before appearance of clinical abnormality will help prevent bilirubin neurotoxicity.

Funding: Nil, Conflict of interest: None initiated, Perission from IRB: Yes

\section{References}

1. Singhal PK, Singh M, Paul VK, et al. Spectrum of neonatal hyperbilirubinemia: an analysis of 454 cases. Indian Pediatr. 1992 Mar;29(3):319-25.

2. Connolly AM, Volpe JJ. Clinical features of bilirubin encephalopathy. Clin Perinatol. 1990 Jun;17(2):371-9.

\section{Original Research Article}

3. Olds C, Oghalai JS. Audiologic impairment associated with bilirubin-induced neurologic damage. SeminFetal Neonatal Med. 2015 Feb;20(1):42-46. doi: 10. 1016/j.siny.2014.12.006. Epub 2015 Jan 7.

4. Smith CM, Barnes GP, Jacobson CA, Oelberg DG. Auditory brainstem response detects early bilirubin neurotoxicity at low indirect bilirubin values. J Perinatol. 2004; 24:730-2.

5. Jiang ZD, Zhang L, Wu YY, et al. Brainstem auditory evoked responses from birth to adulthood: development of wave amplitude. Hear Res. 1993 Jun;68 (1): 35-41.

6. Karmel BZ, Gardner JM, Zappulla RA, Brain-stem auditory evoked responses as indicators of early brain insult. Electroencephalogr Clin Neurophysiol. 1988 Nov-Dec;71(6):429-42

7. American Academy of Pediatrics Subcommittee on Hyperbilirubinemia. Management of hyperbilirubinemia in the newborn infant 35 or more weeks of gestation. Pediatrics. 2004 Jul;114(1):297-316.

8. Oudesluys-Murphy AM, Harlaar J. Neonatal hearing screening with an automated auditory brainstem response screener in the infant's home. Acta Paediatr. 1997 Jun;86(6):651-5.

9. Watchko JF. Neonatal hyperbilirubinemia--what are the risks? N Engl J Med. 2006 May 4;354(18):1947-9. DOI:10.1056/NEJMe068053

10. Shapiro SM. Bilirubin toxicity in the developing nervous system. Pediatr Neurol. 2003 Nov; 29 (5): 410-21.

11. Smitherman H, Stark AR, Bhutani VK. Et al. Early recognition of neonatal hyperbilirubinemia and its emergent management. SeminFetal Neonatal Med. 2006 Jun; 11 (3): 214-24. DOI: 10. 1016/j. siny. 2006. 02. 002.

12. Vohr BR. New approaches to assessing the risks of hyperbilirubinemia. Clin Perinatol. 1990 Jun;17(2): 293-306.

13. Vohr BR, Barry L, Rapisardi G, O'Dea C, Brown L, Peucker $\mathrm{M}$ et al. Abnormal brain stem function (brainstem auditory evoked response) correlates with acoustic cry features in term infants with hyperbilirubinemia. The Journal of Paediatr. 1989 Aug; 115 (2): 303-8. 


\section{Original Research Article}

14. Palmer C, Smith MB. Assessing the risk of kernicterus using nuclear magnetic resonance. Clin Perinatol. 1990 Jun;17(2):307-29.

15. Hansen TW. Bilirubin brain toxicity. J Perinatol. 2001 Dec;21 Suppl1:S48-51; discussion S59-62. DOI: 10.1038/sj.jp.7210634

16. Sharma R, Grover N, Sankhyan N, Sharma ML. Auditory brainstem responses in neonatal hyperbilirubinemia and effect of therapy. Indian J. Otolaryngol Head Neck Surg. 2006 Oct; 58(4):340-42.

17. Rattay F, Danner SM. Peak I of the human auditory brainstem response results from the somatic regions of type I spiral ganglion cells: evidence from computer modeling. Hear Res. 2014 Sep;315:67-79. doi: 10.1016/j. heares.2014.07.001. Epub 2014 Jul 11.

18. Deorari AK, Singh M, Ahuja GK, et al. One year outcome of babies with severe neonatal hyperbilirubinemia and reversible abnormality in brainstem auditory evoked responses. Indian Pediatr. 1994 Aug; 31(8):915-21.

19. Funato M, Teraoka S, Tamai H, et al. Follow-up study of auditory brainstem responses in hyperbilirubinemic newborns treated with exchange transfusion. Acta Paediatr Jpn. 1996 Feb; 38 (1):17-21.
20. Agrawal VK, Shukla R, Misra PK, et al. Brainstem auditory evoked response in newborns with hyperbilirubinemia. Indian Pediatr. 1998 Jun; 35 (6): 513-8.

21. Perlman M, Fainmesser P, Sohmer H, Tamari H, Wax Y, Pevsmer B. Auditory nerve-brainstem evoked responses in hyperbilirubinemic neonates. Pediatrics. 1983; 72(5):658-64. 37.

22. Shapiro SM. Acute brainstem auditory evoked potential abnormalities in jaundiced Gunn rats given sulphonamide. Pediatr Res. 1988; 23(3): 306-10. 38.

23. Shapiro SM. Reversible brainstem auditory evoked potential abnormalities in jaundiced Gunn rats given sulfonamide. Pediatr Res. 1993 Nov; 34(5): 629-33. DOI: 10. 1203/00006450-199311000-00014.

24. Gupta AK, Raj H, Anand NK. Auditory brainstem responses $(\mathrm{ABR})$ in neonates with hyperbilirubinemia. Indian J Pediatr. 1990 Sep-Oct;57(5):705-11.

25. Sharma $\mathrm{P}$, Chhangani $\mathrm{N}$, Meena KR, Jora R, Sharma N, Gupta B. Brainstem evoked response audiometry (BAER) in neonates with hyperbilirubinemia. Indian J Pediatr. 2006; 73(5):413-6.

\section{How to cite this article?}

Kosam A, Khunte M, Panigrahi D. Effect of neonatal hyper bilirubinemia on brain stem auditory evoked response. Int $\mathbf{J}$ Pediatr Res. 2019;6(02):64-69. doi:10.17511/ijpr.2019.i02.03 